\title{
EL SEPTENARIO YÁMBICO DE PLAUTO
}

The iambic septenarius is one of the verse forms most often used by Plautus; he uses it only slightly less frequently than the iambic senarius and the trochaic septenarius. The iambic septenarius underwent a long process of evolution, reaching its most developed form in the work of Plautus who took many liberties with it, e. g. by introducing a wide variety of substitutions and by freely shifting the position of the dieresis. Nonetheless, as this paper tries to demonstrate, it is also subject to certain rules which determine its metrical identity.

La escasa atención de que ha disfrutado tradicionalmente el septenario yámbico quizá se haya debido a la preeminencia del trímetro-senario yámbico, el verso más importante, cuantitativamente, a lo largo de toda la historia del drama grecolatino. Los tratadistas antiguos', desde Varrón, consideraban el tetrámetro yámbico cataléctico como el producto de la unión del trímetro más un monómetro cataléctico, sin tener en cuenta las particularidades del verso largo yámbico.

El autor de tal unión sería, al parecer, Hiponacte, quien ya le dio su carácter cómico; así, no aparece en la tragedia griega, y aun en la comedia está ausente de la parábasis y es ampliamente usado en el agón. Hasta el hallazgo del Dyskolos de Menandro, los pasajes compuestos en tetrámetros pertenecian en su gran mayoría a las comedias de Aristófanes; hoy en día contamos con unos 80 versos bien representativos del verso de la comedia nueva griega, que es la fuente de las comedias de Plauto.

Mario Victorino cuenta que el verso era muy usado por los comediógrafos antiguos: en Roma, Plauto, Cecilio, Turpilio y los autores de atelanas: est enim iocosis motibus emollitum gestibusque agentium satis! accomodatum $^{2}$. De la tragedia romana, parece ser que está ausente: así.

${ }^{\prime}$ Cf. Terenciano Mauro VI p. 396, 2372 K; Diomedes I p. 504, 13 K; M. Victorino VI p. $135,15 \mathrm{~K}$.

2 Cf. M. Victorino, loc. cit., 15. 
opina, entre otros, L. Mueller ${ }^{3}$; ya Varrón lo había llamado quadratus comicus, para oponerlo al septenario trocaico, el tragicus quadratus; sin embargo, el septenario trocaico no es específico de la tragedia, ya que en la comedia plautina es el segundo verso en extensión. L. Strzelecki ${ }^{4}$ se pregunta, entonces, cómo podemos estar seguros de que no hubo septenarios yámbicos trágicos; rastreando entre los fragmentos editados por Ribbeck ${ }^{5}$, el metricólogo polaco encontró tres versos de Accio, más un final de verso del mismo autor, que mediante una ordenación distinta pueden interpretarse como septenarios yámbicos; otro de Pacuvio, y por fin un fragmento anónimo citado por Pseudo-Censorino, de tono trágico, que es con seguridad un ia7.

¿Hubo, entonces, septenarios yámbicos en la tragedia romana? Los datos no son seguros, y la controversia ha sido abundante. De cualquier modo, en Roma el verso, fuera de la comedia arcaica, está presente en algunos fragmentos de las Menippeae de Varrón: B 133-135; 214; $308-9 ; 357 ; 449 ; 576$. Aunque la colometría no es muy segura, en estos versos se advierte ya la vuelta al esquema griego, con la adopción del esquema yámbico puro en los pies pares. El carmen 25 de Catulo nos ofrece, por otra parte, 13 tetrámetros sin elemento bisilábico alguno (bien es verdad que el verso 5 está cruciatus); los siguientes tetrámetros pertenecen ya a Ausonio. Es claro, en cualquier caso, que la vida del septenario, como versión romana del tetrámetro, tiene su apogeo en los aproximadamente 1.300 septenarios yámbicos de Plauto, y acaba en época de Varrón.

El septenario plautino es un verso propio de los cantica, frecuente en largas tiradas que constituyen una escena entera, dialogada entre varios personajes: cf. As. 407-503, Rud. 331-413. En otros casos, precede o sigue a versos igualmente estíquicos, como el senario yámbico y el septenario trocaico; pero no faltan septenarios yámbicos, a veces en escaso número, combinados con ritmos muy diversos: anapésticos, créticos, baquíacos. Contienen un buen número de septenarios las comedias Asinaria, Miles gloriosus y Rudens, con 321, 211 y 205 respectivamente.

Las comedias de Plauto tienen, como es bien sabido, su antecedente en la Comedia Nueva griega; los 73 versos más o menos completos del

${ }^{3}$ De re metrica poetarum latinorum praeter Plautum et Terentium, Petropoli et Lipsiae 1894, p. 107.

${ }^{4}$ De re metrica tragicorum romanorum quaestiones..., I. De septenario iambico eiusque in tragoedia Romana usu.

s Tragicorum romanorum fragmenta, Lipsiae 1897. La misma lectura que Ribbeck da, en todos los casos, E. H. Warmington, Remains of old Latin II, LondresCambridge (Mass.) 1936. 
Dyskolos pueden ser, pues, muy ilustrativos a la hora de establecer una comparación entre la utilización griega y la romana del tetrámetro-septenario; veamos qué caracteriza los versos de Menandro:

1. El séptimo pie, último completo, del tetrámetro menandreo es siempre un yambo; pero ésta es la norma de la que no se apartan los comediógrafos griegos, con la sola excepción del anapesto $7 .^{\circ}$ de Aristófanes en Thesm. $547^{6}$.

2. Los cortes son poco variados: existe solamente la diéresis central, tras el $4 .^{\circ}$ tiempo fuerte, y la cesura un elemento más adelante. La diéresis predomina ligeramente, pues la tienen un $53,42 \%$ de los versos, mientras que la segunda afecta a un $46,57 \%$.

3. No hay hiatos en diéresis ni en cesura, y sólo una sinalefa en diéresis (Dysk. 881).

4. Sustituciones (las que se indican con asterisco no son seguras):

\begin{tabular}{|l|c|c|c|}
\hline & Espondeo & Tribraco & Dáctilo \\
\hline $1 .^{\text {er pie }}$ & $40+5^{*}$ & 1 & $4+2^{*}$ \\
\hline $2 .^{\circ}$ pie & & 4 & \\
\hline $3 .^{\text {er pie }}$ & $35+3^{*}$ & $1+1^{*}$ & 2 \\
\hline $4 .^{\circ}$ pie & & 4 & \\
\hline $5 .^{\circ}$ pie & $38+2^{*}$ & $3+2^{*}$ & $10+1^{*}$ \\
\hline $6 .^{\circ}$ pie & & 11 & \\
\hline Total & $113+10^{*}$ & $24+3^{*}$ & $16+3^{*}$ \\
\hline
\end{tabular}

Es evidente que el espondeo es el pie sustituto más frecuente; dado que sólo los pies impares - y nunca el séptimo - son susceptibles de recibir sustituciones, el porcentaje de espondeos es del 51,59+4,56\%; de dáctilos, pies impuros asimismo, el 10,50 + 0,45\%; los tríbracos, que por ser puros pueden aparecer en cualquier pie, escasean especialmente: sólo un $5,25+0,68 \%$. No hay anapestos.

5. Elementos bisilábicos. Al faltar los anapestos y proceleusmáticos, los elementos resueltos están restringidos a las sedes pares del verso, excluyendo la $14 .^{\text {a }}$, puesto que el $7 .^{\circ}$ pie es siempre yambo: el total

${ }^{6}$ Lo cita F. Perusino en Il tetrametro giambico catalettico nella commedia greca, Roma 1968, p. 77. 
de elementos bisilábicos es de $40+6$, un $54,79+8,21$ por 100 versos. Los elementos más frecuentemente resueltos son el $10 .^{\circ}$, con $13+3$ elementos bisilábicos, y el $12 .^{\circ}$, con 11 . Los que llevan asterisco son sólo probables.

\begin{tabular}{|c|c|}
\hline \multicolumn{2}{|c|}{ Elementos bisilábicos } \\
\hline $2 .^{\circ}$ elemento & $5+2^{*}$ \\
\hline $4 .^{\circ}$ elemento & 4 \\
\hline $6 .^{\circ}$ elemento & $3+1^{*}$ \\
\hline $8 .^{\circ}$ elemento & 4 \\
\hline $10 .^{\circ}$ elemento & $13+3^{*}$ \\
\hline $12 .^{\circ}$ elemento & 11 \\
\hline Total & $40+6^{*}$ \\
\hline$\%$ & $54,79+8,21$ \\
\hline
\end{tabular}

Como vemos, el tetrámetro menandreo es un verso notablemente rígido; las cosas cambiarán en el verso de Plauto.

Entrando ya en terreno latino, hay que hacer algunas precisiones iniciales sobre la cantidad ambigua de ciertas sílabas que en época arcaica pueden considerarse largas o breves; son las siguientes:

- Sílabas finales con $-s$ caduca tras vocal breve. La ambigüedad sobrevive hasta la época de Cicerón, y es Catulo el último poeta que recurre -en un pentámetro-- a la $-s$ caduca ${ }^{7}$. Los autores arcaicos son muy dados a «recuperar» la cantidad breve por naturaleza de una sílaba final en $-s$ y que va seguida de consonante: cf. Pl., As. 731 satǐs iam delusum censeo $\|$; naturalmente, la brevedad de esta sílaba es reconocible porque forma parte de un elemento bisilábico. En cambio, cuando la sílaba constituye por sí misma un tiempo fuerte, la $-s$ traba la vocal: cf. Cist. 307 quamquam uetüs cantherius \|. Los tiempos débiles son los que plantean la incertidumbre: no sabemos si son breves o largos los finales como Most. 164 in pectūs perpluit meum || o Poen. 1228 || nam faxo - mea erīs sponsa.

- Sílabas terminadas en - $t$. En este caso, la duda existe ante inicial vocálica; se trata de sílabas originariamente largas, que se estaban abre-

${ }^{7}$ Cf. Carm. CXVI 8 at fixus nostris tu dabis supplicium. 
viando en vida de Plauto. Obviamente, en tiempo fuerte funcionan como largas, y de ello hay abundantes ejemplos: cf. Mil. 1244 || desiderèt, exspectet., Rud. 390 || possèt: eam ueretur. Sólo un septenario hemos encontrado en el que la sílaba en cuestión haya de considerarse breve, por constituir el $4 .^{\circ}$ tiempo débil en un verso con diéresis: Cist. 312 nimi' lepide $<e x\rangle$ concinnauit hasce $\|$ aedis Alcesimarchus. El verso lo recoge Lindsay ${ }^{8}$, pero no lo cita C. Questa, quien considera largos sin discusión todos estos finales 9 . Para nosotros, hay que considerar ancipitia los que se encuentran en tiempo débil: cf. Rud. 350 recepìt ad se Veneria haec \|, Tru. 252 \| clamore apsterrēt, abigit. Naturalmente, estas premisas se han tenido en cuenta a la hora de elaborar las estadísticas que siguen.

Como decíamos antes, las diferencias entre el verso griego y su derivado romano son importantes:

1. Para empezar, el esquema del septenario a la romana destierra los elementa breuia; únicamente el cuarto pie ha de ser puro en caso de preceder a la diéresis central. Con ello nos encontramos, ya de entrada, con que las sustituciones sólo se verán restringidas, en su número y lugar, por el arbitrio del poeta.

2. El séptimo pie, que ahora es anceps, no tiene por qué estar ocupado por un yambo. Sí es verdad que abundan en él los yambos - más de la mitad (cf. As. 393 \|: quid eius atriēnsis?)-, y que tiene gran tendencia a ser un pie bisilábico: los espondeos son casi una cuarta parte (cf. Mil. $1281 \|$ : :nescioquis eccum incēdit); el resto, dáctilos (cf. As. 719

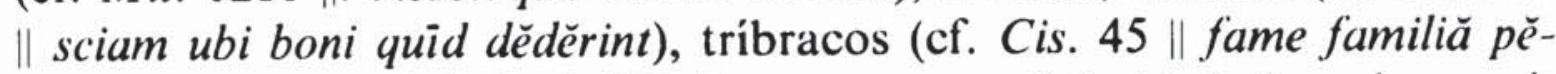
rěat) y anapestos (cf. Rud. 371 || nos uentus exănïmätas). Los dos posibles proceleusmáticos (As. 430 || erus in hara, haud aedibŭs, hăbĭtat y Pers. 290 || male loquere?: tandem ŭtĭ licĕat son muy discutibles, y ya Lindsay, aunque los aceptó en su edición de Plauto, advertía que se debían corregir ${ }^{10}$.

3. El corte del verso se ha estabilizado mucho: la diéresis central alcanza a la inmensa mayoría de los versos, con un porcentaje de entre el 98,5 y el $99 \%$. Esto representa la casi abolición de la cesura tras el $9 .^{\circ}$ elemento, y puede ser interesante la comparación con lo que ocurre en el septenario trocaico de Plauto: alrededor del $95 \%$ de sus $\operatorname{tr} 7$ tienen diéresis central, según datos aportados por J. Soubiran ".

${ }^{8}$ Op. cit., pp. 136 y 323.

${ }^{9}$ Cf. Introduzione alla metrica di Plauto, Bolonia 1967, p. 9.

10 En Early Latin Verse, Oxford 1968 (= 1922), p. 276.

"En Essai sur la versification dramatique des romains. Sénaire iambique et septénaire trochaïque, París 1988. 
La diéresis es, pues, un elemento de sorprendente regularidad; y, sin embargo, su misma existencia da pie a Plauto, y esto es específico suyo, a la posibilidad de sinalefas, hiatos, ancipitia e hiatos con ancipitia ante la diéresis.

Alguna sinalefa en esta sede aparecía ya en Menandro; pero Plauto multiplica los encuentros vocálicos, y no los evita en la diéresis; hay unos 23 casos de ello (cf. Rud. 690 Veneris, quod amplexae modo, und (e) $\|$ abrepta per uim miserae?). Más frecuente, incluso, es el hiato: cf. As. 709 postidea ad pistores dabō $\|$ ut ibi cruciere currens. No falta la sílaba considerada anceps ante la diéresis, cf. Mos. 162 haec illa est tempestas me $\breve{\bar{a}} \|$ mihi quam modestiam omnem. Y, en unos pocos versos, el hiato supone a su vez la consideración del $8 .^{\circ}$ elemento como anceps: cf. As. 613 certum est efficere in me omniä \| eadem quae tu in te faxis.

No siempre es fácil, sin embargo, la determinación de la diéresis; en un grupo de septenarios plautinos podríamos considerar que existe bien cesura o bien diéresis, y otros carecen, aparentemente, de todo corte regular; son los siguientes:
A. Asin. 721
opto annum hunc perpetuom mihi huius operas.:: impetrasti.
Epid. 356
ut quom rediisses ne tibi eiius copia esset.:: eugae!
B. Epid. 361
C. Asin. 469 is adornat, adueniens domi extemplo ut maritus fias.
733
nemo accipit, aufer te domum, apscede hinc, ne male loquatur.
argentum ad té.:: ut temperi opportuneque attulistis.
Mos. 183 quid ais, scelesta? quo modo adiurasti? ita ego istam amarem?
Poen. 1229
ite in ius, ne moramini. antestare me atque duce.
Rud. 366
$<$ de $>$ naui timidae ambae in scapham insiluimus, quia uidemus.
397 credo aliquem immersisse atque eum excepisse.:: id misera maestast.
1322
quid dare uelis qui istaec tibi inuestiget indicetque?

A. Aquellos que podrían tener cesura o diéresis son As. 721 y Ep. 356, dos casos muy similares: se trata de la existencia, en la parte central del verso, de las secuencias mihi huius y tibi eius, susceptibles de dos escansiones distintas: la aparentemente normal, mih(i) huius $\|, \operatorname{tib}(i)$ eius $\|$, y aquella en que se ha de recurrir a una licencia: mihi $\|$ huius, tibi $\|$ eius, con hiato en la diéresis y sinéresis tras ella. No optamos, de momento, por ninguna de las dos, aunque dado que la norma en Plauto es la diéresis, y que abundan licencias como las aquí utilizadas, nos sentimos tentados por la segunda posibilidad.

B. Distinto es lo que ocurre en Ep. 361: aquí tenemos, en el centro del verso, un polisílabo extemplo en sinalefa con la palabra siguiente; es, naturalmente, permisible, por más que rara, la cesura en sinalefa extempl(o) $\| u t$. Sin embargo, veamos cuál es el problema en el resto de los versos citados, $\mathrm{C}$, que a primera vista carecen de corte: en todos 
ellos, tal como en Epid. 361, la palabra que "obstruye» la diéresis es un compuesto: apscede, opportune, extemplo, adiurasti, antestare, insiluimus, excepisse, inuestiget (podría plantearse la duda, en antestare, de si es compuesto de sto o de testor; el contexto indica claramente que se trata del imperativo de an-testor, según Ernout-Meillet ${ }^{12}$ de *ante-testor. Y, también en todos los casos, incluido Ep. 361, la diéresis estaría detrás del preverbio. Todo esto, como es evidente, da mucho que pensar.

4. Veamos ahora en qué número y lugar se presentan las sustituciones; dado que los siete pies son libres de acogerlas - con la restricción del $4 .^{\circ}$ ante cesura - es previsible que se multipliquen. Las estadísticas que sigue han sido elaboradas sobre un muestreo de 300 septenarios yámbicos de Plauto: As. 381 a 480 , Mil. 354 a 410 y 1216 a 1258 , Rud. 290 a 389.

\begin{tabular}{|c|c|c|c|c|c|c|c|c|}
\hline & \multicolumn{2}{|c|}{$P$. puros } & \multicolumn{6}{c|}{ Pies condensados } \\
\cline { 2 - 9 } & $I A$ & $T R$ & $S P$ & $D$ & $A N$ & $P R$ & $I A / S P$ & $T R / D$ \\
\hline $1 .^{\text {er }}$ pie & 40 & 1 & 154 & 46 & 53 & 5 & 1 & - \\
\hline $2 .^{\circ}$ pie & 112 & 12 & 113 & 43 & 12 & - & 5 & 3 \\
\hline $3 .^{\text {er }}$ pie & 34 & 5 & 206 & 20 & 16 & 1 & 10 & 8 \\
\hline $4 .^{\circ}$ pie & 296 & & 4 & & - & & & \\
\hline $5 .^{\circ}$ pie & 54 & 7 & 139 & 29 & 64 & 5 & 2 & - \\
\hline $6 .^{\circ}$ pie & 91 & 9 & 130 & 43 & 8 & 1 & 12 & 6 \\
\hline $7 .^{\circ}$ pie & 173 & 13 & 68 & 11 & 9 & $(1)$ & 24 & 1 \\
\hline Total & 800 & 47 & 814 & 192 & 162 & 13 & 54 & 18 \\
\hline
\end{tabular}

De este cuadro se deducen datos interesantes; en primer lugar, los pies puros son $847+72$, un $40,33+3,42 \%$ del total, y entre ellos son mucho más numerosos los yambos $(800+54)$ que los tríbracos $(47+18)$. En general, pues, hay más pies condensados que puros. Pero ¿es similar el porcentaje de pies condensados en las sedes pares - las que en el verso griego estaban obligadas a contener un pie duro- y en

${ }^{12}$ A. Ernout y A. Meillet, Dictionnaire Etymologique de la Langue Latine. Histoire des mots, París $1979_{5}$. 
las impares, que siempre fueron libres? Los pies pares, $2 .^{\circ}$ y $6 .^{\circ}$, son condensados en $350+26$ ocasiones (dejamos aparte el $4 .^{\circ}$, que es, casi por definición, yambo); los impares, $1 .^{\circ}, 3 .^{\circ}$ y $5 .^{\circ}$ (pues el séptimo, que ya hemos analizado previamente, tiene características especiales), $738+$ 21. El porcentaje es, entonces, de un $58,33+1,23 \%$ de pies condensados pares, y $82+1 \%$ de pies condensados impares: Con lo que resulta que, pese a la libertad de sustituciones, el verso de Plauto conserva una visible diferenciación entre pies pares y pies impares.

Este tipo de confrontación ya la hizo J. Soubiran al estudiar las sustituciones en el senario yámbico y el septenario trocaico ${ }^{13}$; el resultado era similar: los pies impares del senario, y los pares del septenario trocaico, se veian notoriamente favorecidos a la hora de acoger resoluciones, y dejaban traslucir el recuerdo de los versos griegos que fueron sus antecesores.

5. Veamos ahora lo que ocurre con los elementos bisilábicos:

\begin{tabular}{|c|c|}
\hline \multicolumn{2}{|c|}{ Elementos bisilábicos } \\
\hline $1 .^{\text {er }}$ elemento & 58 \\
\hline $2 .^{\circ}$ elemento & 52 \\
\hline $3 .^{\text {er }}$ elemento & 12 \\
\hline $4 .^{\circ}$ elemento & 58 \\
\hline $5 .^{\circ}$ elemento & 17 \\
\hline $6 .^{\circ}$ elemento & 34 \\
\hline $7 .^{\circ}$ elemento & \\
\hline $8 .^{\circ}$ elemento & \\
\hline $9 .^{\circ}$ elemento & 69 \\
\hline $100^{\circ}$ elemento & 41 \\
\hline $11 .^{\circ}$ elemento & 15 \\
\hline $12 .^{\circ}$ elemento & 59 \\
\hline $13 .^{\circ}$ elemento & 10 \\
\hline
\end{tabular}

${ }^{13}$ Essai..., pp. 27-62. 


\begin{tabular}{|c|c|}
\hline \multicolumn{2}{|c|}{ Elementos bisilábicos } \\
\hline $14 .^{\circ}$ elemento & 26 \\
\hline Total & 451 \\
\hline$\%$ & 150,33 \\
\hline
\end{tabular}

Como se ve, las resoluciones se encuentran situadas, preferentemente, en las sedes $9 .^{\mathrm{a}}, 12 .^{\mathrm{a}}, 1 .^{\mathrm{a}}$ y $4 .^{\mathrm{a}}$, con $69,59,58$ y 58 respectivamente. Es lógico que la $1 .^{\text {a }}$ y la $9 .^{\text {a }}$ acojan abundantes elementos bisilábicos, puesto que se encuentran al principio del verso y del segundo hemistiquio; de la $4 .^{\text {a }}$ y la 12 a $^{\text {a }}$ hablaremos un poco más adelante, pues se encuentran sujetas a una norma.

6. Una vez expuestas las libertades de que gozó, en manos de Plauto, el septenario yámbico, pasaremos a recordar las leyes que subyacen bajo ese esquema aparentemente tan permisivo. Acabamos de referirnos a una norma que afecta a los elementos $4 .^{\circ}$ y $12 .^{\circ}$ : es la llamada norma de Meyer. Según ella, en los elementos $4 .^{\circ}$ y $12 .^{\circ}$ del septenario yámbico está prohibido el final absoluto en sílaba larga de polisílabo si el anceps precedente es largo o bisilábico; en consecuencia, tales elementos tienden a no estar ocupados por una sílaba larga; y, a la inversa, caso de estarlo, a ir precedidos de un anceps breve; veamos unos ejemplos de todo esto en el $2 .^{\circ}$ pie: yambo en As. $650 \mid$ primum omnïum; espondeo formado por final de palabra más polisílabo, Mos. $171 \mid$ ut lepi$d \bar{e}$ rês; espondeo en final de palabra no absoluto sino debido a una sinalefa, As. 408 | Libanum in töstrinam, ut iusseram $\|$. En el $6 .^{\circ}$ pie, yambo final en Cis. 367 || nam occasiō. Espondeo compuesto por dos monosílabos en Cur. $507 \|$ prostant, uōs in; espondeo en final por sinalefa, As. 494 || credam hoc àrgèntum ignoto |. La presencia de esta norma -que sin embargo cuenta en Plauto con alrededor de 30 infracciones, cf. Cis. 705 | quis me rĕuŏcat?--, influye, sin duda, en el elevado número de yambos en los pies $2 .^{\circ}$ y $6 .^{\circ}$ (112 y 91 respectivamente), que sólo es inferior al de los pies $4 .^{\circ}$ (puro ante la diéresis) y $7 .^{\circ}$ (tendente a ser yambo).

El tercer pie del septenario yámbico está sujeto, a su vez, a otra norma: la que impide la doble secuencia yámbica en palabras distintas a final del primer hemistiquio, conocida como norma de Bentley-Luchs; el cuadro III muestra que es precisamente este pie el que menos yambos acoge, sólo 34. Las excepciones a esta norma son escasas (cf. As. 419 |qui latera contěrām tua $\|$ ), y las secuencias permitidas son, en cambio, 
las del tipo espondeo + yambo, Cur. 503 cülpänt eum $\|$; anapesto + yambo, Ep. 379 consilins suis \|, etc.

A su vez, la formación de los elementos bisilábicos, tan numerosos $(150,33 \%$, frente al $54,78+8,21 \%$ de Menandro), se encuentra sujeta a determinadas reglas: las sílabas breves se agrupan de dos en dos en palabras pirriquias - égŏ-, a principio de palabra - $\check{o c u ̆ l i s-o ~ e n ~ i n-~}$ terior -impériosus - ; o bien la primera de ellas, al menos, es un monosilabo: ăd ămicum, něc in omnes. Por lo tanto, están prohibidos los elementos bisilábicos finales de polisílabo, del tipo dicěrĕ, y las secuencias de dos breves repartidas entre final de palabra e inicial de otra: multă tỉbi. Bien es verdad que los elementos bisilábicos finales, y los "desgarrados» disfrutan de cierta tolerancia en las sedes $2 .^{\mathrm{a}}$ y $10 \mathrm{a}^{\mathrm{a}}$ : cf. Rud. 290 |omnibŭ' modis qui pauperes, Per. 284 ||ita sum.:.: quid id attǐnĕt ad te?; Rud. 1327 |millě dăbo nummum.:: somnias., Per. ||quidquìd ěrit, recipe te ad me. Ello probablemente es debido a la especial libertad de que gozan el primer pie del verso y el primero del segundo hemistiquio.

En resumen, el septenario yámbico, al igual que el conjunto de los versos yambotrocaicos, se convierte, en manos de Plauto, en un verso sumamente variado, en el que las sustituciones están permitidas en cualquier pie y los elementos bisilábicos se multiplican; bien es verdad que la constancia de la diéresis viene a ser un elemento estabilizador. Pero además, bajo la aparente libertad de un verso largo, capaz, en principio, de dar cabida a combinaciones métricas sin cuento, el septenario conserva de algún modo el recuerdo de la rigidez del tetrámetro: las normas de Meyer y de Bentley-Luchs, el porcentaje más bajo de sustituciones en los pies pares, las limitaciones a los elementos bisilábicos, ponen un cierto freno y sirven de esqueleto al verso.

Rocio Carande Herrero 